Neuroepidemiology 2010;35:91-92

DOI: $\underline{10.1159 / 000314926}$

\section{Validating the Assessment of Comorbidity in Multiple Sclerosis: Another Step towards Comprehensive Health Care}

Katherine B. Knox

Physical Medicine and Rehabilitation, MS Clinic, Saskatoon City Hospital, Saskatoon, Sask., Canada

Horton et al. [1] validate a self-report comorbidity questionnaire in multiple sclerosis (MS) against the MS clinic medical record. They conclude that self-report is a valid method by which to assess comorbidities in MS. They also conclude that further research is needed to determine the questionnaire's ability to predict mortality, disability, health care utilization and quality of life relative to other sources of comorbidity data. The two-centre study (Winnipeg, Canada, and Cleveland, USA) is powered sufficiently to detect substantial agreement between comorbidities with a prevalence of $5 \%$ or higher. They confirm that self-report accurately captured some of the most common comorbidities in minimally to moderately disabled individuals with MS. Agreement with the medical record was moderate to substantial (kappa $>0.51$ ) for depression, hypertension, migraine and hyperlipidaemia. However, several highly prevalent comorbidities which significantly impact quality of life and health care costs are either not included or are under-reported in this cross-sectional study.

Ideally, a valid comorbidity assessment tool would identify comorbidities important to clinicians, health care administrators and patients across the full spectrum of MS disease. The priority of the clinician is to identify in particular modifiable comorbidities which impact quality of life. Health care administrators are interested in comorbidities which have the greatest impact on health care costs. Given that a lower quality of life is associated with increased health care utilization cost in MS, patients, clinicians and administrators may be interested in overlapping comorbidities [2].

Urinary system and sleep disorders are two common comorbidities in the general population that are excluded from the survey. Prevalence estimates in the general adult population are approximately $10 \%$ for restless leg syndrome [3, 4], 4\% for sleep apnoea/hypopnoea syndrome [5] and $25-45 \%$ for urinary incontinence in women [6]. In the MS population, poor sleep and urinary incontinence have been shown to be significantly and independently associated with lower quality of life [7, 8]. The symptoms related to sleep and urinary disorders may be difficult to differentiate from symptoms related to MS. The challenge of separating MS symptoms from other comorbidity symptoms may be partly overcome by the design of the questionnaire. For example one could ask: 'has a doctor ever told you that you have a sleep disorder in addition to your MS?' On the other hand, how important is it to differentiate the aetiology of these particular symptoms if the final goal is to evaluate quality of life and health care costs? Symptoms related to urinary and sleep dysfunction have a significant impact on quality of life and health care costs [7, 9, 10] - possibly in addition to MS alone. Every effort should be made to include urinary and sleep disorders in a comorbidity questionnaire.

The frequency of reported arthritis (a leading cause of activity restrictions in the general population) is lower in this study compared to that expected in both the MS and the general population [11]. The total frequency for all musculoskeletal conditions was only $11.9 \%$ from the questionnaire data and $6.2 \%$ from medical records [1]. These numbers are derived by totalling arthritis, joint replacements, fibromyalgia and rheumatoid arthritis. In comparison, Warren et al. [8] described a $25.6 \%$ frequency of self-reported arthritis in MS. This is comparable to the estimated frequency of self-reported physician-diagnosed arthritis in the general population of greater than $21 \%$ [11].

The authors themselves address the most significant explanation for differences in the frequencies of self-reported arthritis: chronic and less clearly defined conditions are less likely to be accurately self-reported. However, this does not fully explain the low frequency reported in the medical record [1]. Arthritis prevalence increases significantly with age, with half of adults over the age of 65 reporting arthritis in the general population [11]. The mean age of subjects in the study by Warren et al. [8] was only slightly older than in the study by Horton et al. [1]: 48.7 years and 46.5 years, respectively. Disability levels, which were not available in the study by Warren et al. [8], may also account for differences in arthritis prevalence. Musculoskeletal comorbidity has been associated with an increased odds of severe disability compared to mild disability at the time of MS diagnosis (OR 1.81, 95\% CI 1.252.63) [12]. However, Turpin et al. [13] found a prevalence of muscle, bone or joint problems in MS as high as 54.5\% in minimally disabled subjects (mean age 38.9 years). The specific language utilized (degenerative arthritis vs. bone and joint problems), disability level, and age are among some of the factors to consider when comparing self-reported prevalence rates for arthritis across studies. A study confirming that arthritis prevalence increases in a more severely disabled and older MS sample would further validate the self-report questionnaire proposed by Horton et al. [1].

It is a limitation that subjects with severely impaired upper extremity function, or significant cognitive or physical disability were excluded from the study. If we understood more clearly the frequency of comorbidities across disability, we may be able to implement preventative comorbidity treatment strategies more effectively. For more significantly disabled people, the measurement tool could be filled out by a close caregiver. This data could be compared to other sources of comorbidity data for validity.

The authors explain that a self-report questionnaire is a practical method to capture comorbidity in MS when there are multiple health care providers and comorbidities involved. They ar-

\section{KARGER}

Fax +41613061234 E-Mail karger@karger.ch www.karger.com (c) 2010 S. Karger AG, Basel

0251-5350/10/0352-0091\$26.00/0 
gue that a self-report questionnaire may be better than other comorbidity data sources at identifying modifiable comorbidities [1]. These points are relevant since the treatable or preventable comorbidities are among those important to identify efficiently. Also of high interest are the comorbidities that have the greatest impact on quality of life and health care costs. For these reasons it is reassuring that hypertension, depression and migraine were accurately reported by the questionnaire. Questionnaire-reported comorbidity data may be utilized in the future to better direct patient care and to make meaningful predictions on health care costs and quality of life in MS. These predictions may be improved if sleep and urinary comorbidities are reliably included, and also if those people with more severe disability are represented. The inclusion of comorbidity as part of a comprehensive populationbased approach to MS care is a welcome and needed area of clinical research focus [14].

\section{References}

1 Horton M, Rudick RA, Hara-Cleaver C, Marrie RA: Validation of a selfreport comorbidity questionnaire for multiple sclerosis. Neuroepidemiology 2010;35:83-90.

-2 McCrone P, Heslin M, Knapp M, Bull P, Thompson A: Multiple sclerosis in the UK: service use, costs, quality of life and disability. Pharmacoeconomics 2008;26:847-860.

-3 Ondo WG: Restless leg syndrome (review). Neurologic Clinics 2009;27: 779-799.

4 Gomez-Choco MJ, Iranzo A, Blanco Y, Graus F, Santamaria J, Saiz A: Prevalence of restless legs syndrome and REM sleep behaviour disorder in multiple sclerosis. Mult Scler 2007;13:805-808.

5 Jennum P, Riha RL: Epidemiology of sleep apnoea/hypopnoea syndrome and sleep disordered breathing. Eur Respir J 2009;33:907-914.

6 Milsom I: Lower urinary tract symptoms in women. Curr Opin Urol 2009;19:337-341.
-7 Merlino G, Fratticci L, Lenchig C, Valente M, Cargnelutti D, Picello M, Serafini A, Dolso P, Gigli GL: Prevalence of 'poor sleep' among patients with multiple sclerosis: an independent predictor of mental and physical status. Sleep Med 2009;10:26-34.

8 Warren SA, Turpin KV, Pohar SL, Jones CA, Warren KG: Comorbidity and health-related quality of life in people with multiple sclerosis. Int J MS Care 2009;11:6-16.

-9 Irwin DE, Mungapen L, Milsom 1, Kopp Z, Reeves P, Kelleher C: The economic impact of overactive bladder syndrome in six Western countries. BJU Int 2009;103:202-209.

10 Hall SA, Link CL, Tennstedt SL, Mollon P, Aiyer LP, Chapple CR, Wein AJ, Rosen RC: Urological symptom clusters and health-related qualityof-life: results from the Boston Area Community Health Survey. BJU Int 2009; 103:1502-1508.

11 Helmick CG, Felson DT, Lawrence RC, Gabriel S, Hirsch R, Kwoh CK, Liang MH, Kremers HM, Mayes MD, Merkel PA, Pillemer SR, Reveille JD, Stone JH: National Arthritis Data Workgroup. Estimates of the prevalence of arthritis and other rheumatologic conditions in the United States. Part 1. Arthritis Rheum 2008;58:15-35.

-12 Marrie RA, Horwitz R, Cutter G, Tyry T, Campagnolo D, Vollmer T: Comorbidity delays diagnosis and increases disability at diagnosis of MS. Neurology 2009;72:117-124.

13 Turpin KV, Carroll LJ, Cassidy JD, Hader WJ: Deterioration in the health-related quality of life of persons with multiple sclerosis: the possible warning signs. Mult Scler 2007;13:1038-1045.

14 Metz L: Time for a population based approach to MS Care. Can J Neurol Sci 2007;34:120.

Assist. Prof. Katherine B. Knox, MD, FRCPC

Physical Medicine and Rehabilitation, MS Clinic

Saskatoon City Hospital

Saskatoon, SK S7K 0M7 (Canada)

Tel. +1 306655 8447, Fax +1 3066558404

E-Mail katherine.knox@ saskatoonhealthregion.ca 